\title{
Salud bucal en Chile, situación actual y desafíos futuros
}

\section{Nota Científica}

\author{
Ricardo Cartes-Velásquez 1,2,a. \\ 1 Universidad Andrés Bello, Facultad de Odontología, \\ Concepción, Chile. \\ ${ }^{2}$ Fundación Kimntrum, Concepción, Chile. \\ a Doctor en Ciencias Médicas.
}

\section{Correspondencia:}

Ricardo Cartes-Velásquez: cartesvelasquez@gmail.com Beltrán Mathieu \#7, Concepción, Chile.

ORCID: 0000-0001-5831-7324

\section{Editora invitada:}

Lita Ortiz-Fernández

Universidad Nacional Mayor de San Marcos, Perú.

\section{Oral health in Chile, current situation and future challenges}

Conflicto de intereses: el autor declara no tener conflictos de interés.

Fuente de financiamiento: autofinanciado.

Recibido: 09/03/20

Aceptado: 25/03/20

Publicado: 09/05/20

\begin{abstract}
Resumen
Chile ha visto una mejora en sus indicadores de morbilidad bucal en las últimas décadas, esto ha ido de la mano con la ampliación de las coberturas en promoción, prevención y tratamiento odontológico, priorizando los grupos infantiles, embarazadas y adultos mayores. A pesar de esto, persiste una morbilidad bucal muy alta, especialmente en la población de 20 a 60 años, que tienen escaso acceso a atención odontológica restauradora. El Ministerio de Salud ha propuesto un ambicioso Plan Nacional de Salud Bucal 20182030 que busca mejorar el estado de salud bucal y reducir las desigualdades, esto se basa en los conceptos de equidad, intersectorialidad, participación ciudadana e inclusión y no discriminación, y materializa en cuatro ejes estratégicos: promoción de salud y prevención de enfermedades bucales; provisión de servicios odontológicos de calidad; desarrollo del recurso humano; e investigación, monitoreo y evaluación.
\end{abstract}

Palabras clave: Salud bucal; Política pública; Chile; Caries dental; Equidad (fuente: DeCS BIREME).

\begin{abstract}
In recent decades, Chile has seen an improvement in its indicators of oral morbidity; that has gone hand in hand with the expansion of coverage in promotion, prevention and dental treatment, prioritizing children, pregnant women and the elderly. Albeit this effort, a very high oral morbidity persists, especially in the population aged 20 to 60 years old, whom have little access to restorative dental care. The Ministry of Health has proposed an ambitious 2018-2030 National Oral Health Plan that pursues the improvement of oral health as well as to reduce inequalities, based on the concepts of equity, intersectionality, citizen participation and inclusion and non-discrimination, thus it materializes in four strategic axes: health promotion and prevention of oral diseases; delivery of quality dental services; human resources development; and research, monitoring and evaluation.
\end{abstract}

Keywords: Oral Health; Public policy; Chile; Dental caries; Equity (source: MeSH NLM).

(c) Los autores. Este artículo es publicado por la revista Odontología Sanmarquina de la Facultad de Odontología, Universidad Nacional Mayor de San Marcos. Este es un artículo de acceso abierto, distribuido bajo los términos de la licencia Creative Commons Atribucion - No Comercia_Compartir Igual 4.0 Internacional. (http://creativecommons.org/licenses/by-nc-sa/4.0/) que permite el uso no comercial, distribución y reproducción en cualquier medio, siempre que la obra original sea debidamente citada. 


\section{Introducción}

Por más de 20 años, Chile ha sido visto como un modelo a seguir para el resto de los países latinoamericanos, no solo por el auge económico, sino también por la estabilidad de su democracia, sus buenos indicadores sanitarios, entre otros ${ }^{1}$.

Sin embargo, en octubre de 2019, una serie de protestas iniciadas por estudiantes secundarios en el Metro de Santiago, la capital del país, se esparcieron al resto del territorio nacional. Ya no era la indignación de un pequeño grupo de personas por el alza de 30 pesos chilenos (unos 40 centavos de dólar) en el pasaje del Metro, sino la de la mayoría de la población chilena por una serie de injusticias y desigualdades transversales a todos los planos de la vida diaria durante los últimos 30 años ${ }^{2}$.

Trabajo, educación, vivienda y, por supuesto, salud son algunas de las áreas donde existen al menos dos países o realidades en un solo territorio nacional ${ }^{2}$. Una realidad es comparable a la de los países más ricos del mundo, donde el acceso a viviendas y espacios comunitarios de alto estándar es la norma. Lo mismo ocurre con el acceso a educación con instalaciones y profesores de excelencia, incluso con la posibilidad cierta de realizar pasantías o continuar estudios de postgrado en las mejores universidades del mundo. Otra realidad es comparable a los países del África subsahariana, aquí el acceso a la vivienda y espacios comunitarios de calidad es escaso, persistiendo muchas zonas con campamentos sin condiciones mínimas de salubridad o afectadas por el flagelo de la drogadicción y la violencia ${ }^{3,4}$. Igual situación encontramos en el sistema educativo chileno, uno de los más segregados a nivel internacional, con escuelas sin implementación ni profesores capacitados para entregar una educación adecuada, especialmente si consideramos que a estas escuelas asisten alumnos que vienen de condiciones de deprivación sociocultural ${ }^{5}$.

Por tanto, al analizar la situación de la salud bucal en Chile, debemos considerar lo descrito previamente. El objetivo de esta revisión es describir la situación epidemiológica, las políticas públicas y los desafíos de la salud bucal en Chile.

\section{Sistema de salud chileno}

El sistema chileno es caracterizado por ser uno de provisión mixta, público y privado, tanto a nivel del aseguramiento o financiamiento, como de la prestación o provisión de los servicios sanitarios propiamente tal ${ }^{2}$. Alrededor de 4 de cada 5 chilenos se atiende en el sistema público, a nivel de financiamiento cerca del $80 \%$ están cubiertos por el Fondo Nacional de Salud (FONASA) y por sistemas de salud para las distintas ramas de las fuerzas armadas. Mientras que poco menos del $20 \%$ están cubiertos por las Instituciones de Salud Previsional (ISAPRE), actualmente existen seis ISAPRE abiertas y seis cerradas, estas últimas otorgan prestaciones a trabajadores de una institución en particular, mientras que las primeras están abiertas a cualquier persona ${ }^{6}$.
Nominalmente, el 7\% de los sueldos de los trabajadores se destina o cotiza en FONASA o ISAPRE para financiar los servicios de salud, pero en el caso de quienes están cubiertos por FONASA el Estado complementa ese $7 \%$ con recursos propios y en el caso de las personas cubiertas por las ISAPRE se deben acoger a un plan de salud que puede costar más del 7\% de su sueldo, por lo que debe pagar esa diferencia aumentando el porcentaje ${ }^{6,7}$.

Es importante tener en cuenta que la cobertura de financiamiento en Chile no es totalmente universal, sino que se organiza por tramos o planes. En el caso de FONASA existen cuatro tramos: A, B, C y D; estos se organizan según los niveles de ingreso de los cotizantes, mientras el tramo A está destinado a personas indigentes o carentes de recursos, el tramo B es para personas con ingresos menores a \$250.000.- pesos chilenos (unos 300 dólares), el tramo C para personas con ingresos entre $\$ 250.000$. - y $\$ 365.000$.- pesos chilenos (unos 440 dólares) y el tramo $\mathrm{D}$ para personas con ingresos superiores a esta última cifra. Las personas en los tramos A y B tiene acceso a gratuidad en el sistema público (hospitales y consultorios), mientras que las personas del tramo $\mathrm{C}$ y $\mathrm{D}$ tienen una bonificación del $90 \%$ y $80 \%$, respectivamente, por lo que deben realizar un copago para atenderse en el sistema público ${ }^{8}$. Esto generalmente ocurre en la atención con especialistas en hospitales de mayor complejidad, ya que en consultorios u hospitales de menor complejidad o rurales, la atención es casi siempre gratuita.

Por otro lado, las personas que optan por cotizar en ISAPRE tienen coberturas en función del plan, a mayor cobertura, el plan tiende a ser más caro. Lo mismo ocurre con "grupos de riesgo", mientras hombres jóvenes pueden acceder a planes de ISAPRE relativamente baratos y con buenas coberturas (copago cero para la mayoría de los servicios o prestaciones de salud), mujeres en edad fértil, adultos mayores o personas con enfermedades de alto costo solo pueden acceder a planes de ISAPRE de alto costo y con coberturas menores (existen copagos altos) ${ }^{6,7}$. Esta situación ha sido motivo de críticas en los últimos años, por lo que se han propuesto otros modelos de aseguramiento que promuevan una mayor equidad y solidaridad del sistema ${ }^{9}$. Por último, en el caso de las fuerzas armadas, la Caja de Previsión de Fuerzas Armadas y de Orden financia la atención en salud con impuestos generales ${ }^{6}$.

En el caso de la prestación de servicios de salud, también existe una parte pública y otra privada. En el caso del sector privado existen prestadores institucionales donde se incluyen desde pequeños centros médicos a grandes clínicas, mientras que también hay prestadores individuales que pueden prestar sus servicios en consultorios propios o a través de los grandes prestadores. En tanto que, en el sistema público encontramos solo prestadores institucionales que están organizados en dos niveles de administración ${ }^{6}$. La atención primaria de salud pública tiene como establecimiento de salud principal al Centro de Salud Familiar (CESFAM), los que son de administración municipal en casi su totalidad. Cada CESFAM 
cuenta con grupos multidisciplinarios de profesionales que atienden a un sector de la comunidad ${ }^{10}$. A modo de ejemplo, una comuna de 20000 habitantes tiene un CESFAM que se organiza en dos sectores, cada sector tiene su propio equipo de cabecera con médico, enfermera, dentista y matrona, mientras que otros profesionales como nutricionistas, kinesiólogos, farmacéuticos y otros, pueden estar asignados a un sector determinado o ser transversales para la atención de toda la comunidad o un grupo determinado (niños, embarazadas, pacientes con patologías crónicas, etc.). Por otro lado, el nivel secundario y terciario de atención cuenta con hospitales de diversa complejidad y son administrados por alguno de los 29 servicios de salud a lo largo de Chile, con excepción de los autogestionados. Cada hospital cuenta con una oferta variable de especialidades y servicios de apoyo, a los que normalmente se accede por derivación desde el nivel de atención primario ${ }^{10}$.

En Chile, aseguramiento y provisión no están atados, por lo que una persona asegurada por FONASA puede acceder a atención privada y viceversa. En el caso de FONASA, esto se expresa en la existencia de dos modalidades de atención: la atención institucional (MAI) y la libre elección (MLE). La MAI se entrega en consultorios y hospitales públicos, es gratis para el tramo $\mathrm{A}$ y $\mathrm{B}$, mientras que tiene un copago para los tramos $\mathrm{C}$ y D. La MLE es entregada por prestadores privados en convenio con FONASA, en este caso la persona debe comprar un bono que cubre la diferencia entre el precio cobrado por el prestador y lo que cubre FONASA. En caso de ISAPRE, los cotizantes pueden elegir atenderse en centros públicos y privados, pero generalmente se atienden en los últimos ${ }^{6}$.

Además de lo anterior, es importante agregar lo que ha significado el programa de Garantías Explicitas en Salud (GES) en el sistema de salud chileno. El GES incluye una serie de beneficios para las personas afiliadas a FONASA o ISAPRE que sufran de alguna enfermedad o condición incluida en el GES, beneficios que están garantizados por ley. Estas garantías incluyen: Acceso: derecho por Ley de la prestación de Salud; Oportunidad: tiempos máximos de espera para el otorgamiento de las prestaciones; Protección financiera: la persona beneficiaria cancelará un porcentaje de la afiliación; y Calidad: otorgamiento de las prestaciones por un prestador acreditado o certificado. Cuando se lanzó el GES en 2006, se cubrieron 56 patologías, pero actualmente son 85 patologías las que tienen estas garantías ${ }^{11}$. A pesar del avance que significa el GES, no ha estado exenta de críticas respecto a cuáles son las patologías que se incluyen y qué sucede con otras enfermedades fuera del régimen GES, las que no tienen garantizada de igual manera su atención, con la consiguiente pérdida de calidad de vida para quienes las sufren. Un sistema similar al GES es el sistema que implementó la Ley 20.850 para asegurar el financiamiento de diagnósticos y tratamientos basados en medicamentos, dispositivos médicos y alimentos de alto costo con efectividad probada, sus beneficiarios son todas las personas que tienen un Sistema de Salud Previsional en
Chile. Actualmente existen 27 patologías cubiertas por este sistema ${ }^{12}$.

Por último, es también importante mencionar que el sistema público de salud cuenta con una serie de orientaciones o metas que privilegian la atención de ciertos grupos de personas. Uno de los más importantes son los Indicadores de Actividad de la Atención Primaria Municipal que están ligados a objetivos sanitarios esenciales que son de amplia cobertura, como mantener un porcentaje mínimo de las personas con diabetes tipo 2 o con hipertensión bajo control, asegurar un temprano control del embarazo o exámenes de salud preventiva ${ }^{13}$.

\section{Políticas en salud bucal en Chile}

Tal como señala el Ministerio de Salud de Chile (MINSAL), "la Política de Salud Bucal está orientada a la prevención y promoción de la Salud Bucal de la población, con énfasis en los grupos más vulnerables. Considera además actividades recuperativas en grupos priorizados mediante acciones costo efectivas basadas en la mejor evidencia disponible" ${ }^{14}$. Actualmente esto se materializa en el Plan Nacional de Salud Bucal 2018-2030 propuesto por el Departamento de Salud Bucal del MINSAL, que señala "El Plan Nacional de Salud Bucal se estructura en 4 ejes estratégicos (EE), 12 objetivos estratégicos (OE), 34 estrategias (E) y 70 metas y resultados esperados de responsabilidad del nivel central o regional. Las metas se definen a corto plazo (2020), mediano (2025) y largo plazo (2030) y constituyen la base para la formulación de los Planes Operativos Anuales de las SEREMIs, Servicios de Salud y Departamentos o Unidades de distintas Divisiones de ambas Subsecretarias del Ministerio de Salud" 15.

El objetivo nacional de este Plan es mejorar el estado de salud bucal y reducir las desigualdades injustas, esto se basa en los conceptos de equidad, intersectorialidad, participación ciudadana e inclusión y no discriminación. A su vez, los cuatro ejes estratégicos corresponden a: promoción de salud y prevención de enfermedades bucales; provisión de servicios odontológicos de calidad; desarrollo del recurso humano; e investigación, monitoreo y evaluación. En la Tabla se muestran los objetivos estratégicos con sus respectivas metas y estrategias ${ }^{15}$.

Este ambicioso Plan es resultado de 40 años de evolución desde las primeras políticas en salud bucal formuladas por el MINSAL en 1978, que desde un principio privilegiaron niños, niñas y adolescentes (NNA) desde un enfoque preventivo y curativo. Luego en las décadas de 1980 y 1990 se agrega como grupo priorizado a las embarazadas, se inicia la fluoración del agua potable a nivel nacional alcanzando al $83 \%$ de la población urbana (solo la región del Biobío no cuenta con fluoración), se comenzó con la atención dental de adultos mayores y mujeres jefas de hogar, especialmente con rehabilitación protésica, y se reforzó el componente preventivo con la inclusión de la salud bucal en los controles de niño sano ${ }^{15}$.

La década 2000-2010 se caracterizó por la inclusión de la salud bucal en los Objetivos Sanitarios del MINSAL, 
Tabla. Objetivos estratégicos y metas del Plan Nacional de Salud Bucal 2018-2030, Chile

\begin{tabular}{|c|c|c|}
\hline Objetivo estratégico & Meta & Estrategias \\
\hline $\begin{array}{l}\text { Incentivar la valoración y } \\
\text { la alfabetización en salud } \\
\text { bucal. }\end{array}$ & $\begin{array}{l}\text { Nivel de alfabetización en } \\
\text { salud bucal de la población } \\
\text { evaluada }\end{array}$ & $\begin{array}{l}\text { - Aumentar la cobertura de las estrategias de promoción de salud del } \\
\text { modelo de intervención, en el ámbito de acción en Educación } \\
\text { Incorporar al modelo de intervención el ámbito de acción en la } \\
\text { comunidad } \\
\text { - } \quad \text { Aumentar la difusión de la política, programas y garantías en salud } \\
\text { bucal } \\
\text { Evaluar el nivel de alfabetización en salud bucal de la población. }\end{array}$ \\
\hline $\begin{array}{l}\text { Fortalecer la prevención de } \\
\text { enfermedades bucales en el } \\
\text { curso de vida }\end{array}$ & $\begin{array}{l}90 \% \text { de la población chilena } \\
\text { tiene una o más medidas } \\
\text { preventivas para las enfer- } \\
\text { medades bucales. }\end{array}$ & $\begin{array}{l}\text { - Aumentar la cobertura de programas de fluoración masiva } \\
\text { - Aumentar la cobertura de estrategias de prevención de enfermeda- } \\
\text { des bucales del modelo de intervención en el ámbito de acción en } \\
\text { Educación } \\
\text { Incorporar estrategias preventivas al ámbito de acción en la Comu- } \\
\text { nidad. }\end{array}$ \\
\hline $\begin{array}{l}\text { Avanzar en Salud Bucal en } \\
\text { todas las Políticas y Partici- } \\
\text { pación Ciudadana }\end{array}$ & $\begin{array}{l}\text { Mesa Nacional intersectorial } \\
\text { de Salud Bucal con trabajo } \\
\text { en el nivel de Integración. }\end{array}$ & $\begin{array}{l}\text { - Fortalecer las Mesas Intersectoriales de Salud Bucal nacional, regio- } \\
\text { nal y comunal } \\
\text { - } \quad \text { Fortalecer los recursos compartidos intersectoriales } \\
\text { - Fortalecer mecanismos de participación ciudadana a nivel regional. }\end{array}$ \\
\hline $\begin{array}{l}\text { Asegurar la calidad de los } \\
\text { servicios odontológicos. }\end{array}$ & $\begin{array}{l}80 \% \text { de los usuarios de los } \\
\text { programas de salud bucal } \\
\text { priorizados se considera } \\
\text { satisfecho con la calidad de } \\
\text { los servicios odontológicos. }\end{array}$ & $\begin{array}{l}\text { - Actualizar la normativa relacionada con la protección de la calidad } \\
\text { de la atención y seguridad del paciente durante la atención odonto- } \\
\text { lógica } \\
\text { Incorporar la evidencia científica en la toma de decisión relacionada } \\
\text { con la provisión de servicios odontológicos } \\
\text { Incorporar la evaluación de calidad en la provisión de servicios } \\
\text { odontológicos. }\end{array}$ \\
\hline $\begin{array}{l}\text { Avanzar en acceso y co- } \\
\text { bertura universal en salud } \\
\text { bucal. }\end{array}$ & $\begin{array}{l}75 \% \text { de los centros de } \\
\text { atención primaria de salud } \\
\text { con al menos un } 60 \% \text { de } \\
\text { personas menores de } 20 \\
\text { años bajo control. }\end{array}$ & $\begin{array}{l}\text { - Aumentar la cobertura de población bajo control con enfoque de } \\
\text { riesgo odontológico } \\
\text { - Fortalecer programas de salud bucal con enfoque de factores comu- } \\
\text { nes de enfermedades crónicas y curso de vida } \\
\text { - } \quad \text { Aumentar la efectividad de la educación para la salud bucal } \\
\text { Aumentar la cobertura y capacidad de diagnóstico precoz y trata- } \\
\text { miento oportuno de enfermedades bucales. }\end{array}$ \\
\hline $\begin{array}{l}\text { Fortalecer los servicios } \\
\text { odontológicos en las redes } \\
\text { integradas. }\end{array}$ & $\begin{array}{l}80 \% \text { de los Servicios } \\
\text { de Salud tienen centros } \\
\text { odontológicos de especia- } \\
\text { lidades ambulatorias en } \\
\text { funcionamiento según } \\
\text { estándar ministerial. }\end{array}$ & $\begin{array}{l}\text { - } \quad \text { Fortalecer la referencia y contra referencia para las especialidades } \\
\text { odontológicas } \\
\text { - } \quad \text { Reducir la brecha de recursos físicos y equipamiento odontológico } \\
\text { - } \quad \text { Desarrollar redes de especialidades odontológicas. }\end{array}$ \\
\hline $\begin{array}{l}\text { Fortalecer el desarrollo de } \\
\text { recurso humano odontoló- } \\
\text { gico competente de acuerdo } \\
\text { a la necesidad del país }\end{array}$ & $\begin{array}{l}70 \% \text { del recurso humano } \\
\text { odontológico formado de } \\
\text { acuerdo al perfil definido. }\end{array}$ & $\begin{array}{l}\text { Desarrollar recurso humano odontológico de acuerdo al perfil de } \\
\text { desempeño que el país necesita } \\
\text { Actualizar la normativa que regula las competencias del recurso } \\
\text { humano odontológico } \\
\text { - Fortalecer el plan de capacitación continua en salud bucal }\end{array}$ \\
\hline $\begin{array}{l}\text { Mejorar la dotación de } \\
\text { recurso humano odontoló- } \\
\text { gico de acuerdo a estándar } \\
\text { definido. }\end{array}$ & $\begin{array}{l}75 \% \text { de los establecimien- } \\
\text { tos de atención primaria y } \\
\text { secundaria cumple con el } \\
\text { estándar de } \\
\text { recurso humano odontoló- } \\
\text { gico definido. }\end{array}$ & $\begin{array}{l}\text { - Mejorar la relación de horas de recurso humano odontológico de } \\
\text { nivel primario por población } \\
\text { Mejorar la relación de horas de recurso humano odontológico de } \\
\text { especialidad. }\end{array}$ \\
\hline $\begin{array}{l}\text { Actualizar el plan de for- } \\
\text { mación de recurso humano } \\
\text { odontológico de especia- } \\
\text { lidad. }\end{array}$ & $\begin{array}{l}\text { Plan de formación de } \\
\text { recurso humano odontoló- } \\
\text { gico especialista incorpora } \\
\text { estudio de brecha. }\end{array}$ & $\begin{array}{l}\text { - Alinear el plan de formación de recurso humano con el diagnóstico } \\
\text { de brecha } \\
\text { Redefinir las vías de ingreso al programa de formación de especia- } \\
\text { listas. }\end{array}$ \\
\hline $\begin{array}{l}\text { Instalar un sistema de vigi- } \\
\text { lancia del estado de salud } \\
\text { bucal de la población y su } \\
\text { impacto en } \\
\text { la calidad de vida }\end{array}$ & $\begin{array}{l}\text { Diagnóstico nacional de } \\
\text { salud bucal por curso de } \\
\text { vida, realizado por } \\
\text { odontólogo a través de } \\
\text { encuesta poblacional, } \\
\text { actualizado. }\end{array}$ & $\begin{array}{l}\text { - Impulsar convenios de colaboración con instituciones de educación } \\
\text { superior para realizar estudios en salud bucal } \\
\text { - } \quad \text { Actualizar el diagnóstico nacional de salud bucal } \\
\text { Mejorar el análisis de la información existente de estudios y registros } \\
\text { clínicos. }\end{array}$ \\
\hline $\begin{array}{l}\text { Mejorar la calidad, relevan- } \\
\text { cia y disponibilidad de la } \\
\text { información de salud bucal. }\end{array}$ & $\begin{array}{l}\text { Sistema de reporte de } \\
\text { indicadores de salud bucal } \\
\text { instalado. }\end{array}$ & $\begin{array}{l}\text { - Avanzar en la disponibilidad de información de calidad para la toma } \\
\text { de decisión en salud bucal } \\
\text { Generar un sistema de monitoreo y evaluación del Plan Nacional de } \\
\text { Salud Bucal. }\end{array}$ \\
\hline $\begin{array}{l}\text { Mejorar la evaluación y el } \\
\text { monitoreo de los programas } \\
\text { de salud pública. }\end{array}$ & $\begin{array}{l}\text { Cobertura de programas } \\
\text { priorizados considera los } \\
\text { Determinantes Sociales de } \\
\text { la Salud. }\end{array}$ & $\begin{array}{l}\text { Evaluar la cobertura de los programas de salud bucal considerando } \\
\text { los Determinantes Sociales } \\
\text { Fortalecer el componente de evaluación en el diseño de programas } \\
\text { de salud bucal. }\end{array}$ \\
\hline
\end{tabular}


pero en términos prácticos no se realizaron grandes cambios, permaneciendo la priorización de NNA y embarazadas, aunque ampliando la cobertura real para tratamientos curativos. En esta década se ampliaron las estrategias preventivas a todos los menores de 20 años, se incluyó la atención de adolescentes de 12 años como meta sanitaria, y se inició un programa preventivo y promocional dirigido a preescolares que asisten a jardines infantiles (actual Programa Sembrando Sonrisas). Esta década es la del GES, donde también se considera la atención de patologías odontológicas, actualmente son cuatro: Salud oral integral de la embarazada, Salud oral integral para nińos de 6 años, Urgencia odontológica ambulatoria y Salud oral integral a los 60 ańos. A estas cuatro podemos agregar el componente odontológico de la atención de pacientes con fisura labiopalatina ${ }^{15,16}$.

Durante la última década, 2010-2020, ha persistido la priorización de los grupos ya mencionados. Se ha fortalecido el rol del Programa Sembrando Sonrisas para preescolares, se amplió la atención de mujeres mayores de 20 años a través del Programa Más Sonrisas para Chile, se instauró el Programa de Mejoramiento del Acceso a la Atención Odontológica para los Jóvenes de Cuarto Año de Educación Media como una forma de garantizar una buena salud bucal al término de la educación obligatoria (en Chile son 8 años de educación básica y 4 de media), se mejoró el acceso a población adulta trabajadora a través del Programa de Atención de Morbilidad Odontológica que generalmente entrega atención en horario vespertino (luego de la jornada laboral ordinaria), así como a población pobre mediante el Programa de Hombres de Escasos Recursos, y FONASA agregó acceso odontológico en MLE para adolescentes de 12 a 17 años, quienes pueden acceder a tratamientos preventivos y curativos entregados por prestadores privados mediante un Pago Asociado a Diagnóstico (PAD). En 2017 se creó el Programa Control con Enfoque de Riesgo Odontológico (CERO) en el que se realiza la mantención de población bajo control desde los 6 meses hasta los 7 años de edad según la categorización del riesgo, de manera que el escolar llegue a su atención al GES Odontológico de 6 años sin caries $15,17,18$.

Un aspecto relevante planteado en el tercer objetivo estratégico del Plan 2018-2030 guarda relación con el recurso humano. Acá es importante mencionar que la política del MINSAL se enfoca en la formación de especialistas mediante la Etapa de Destinación y Formación (EDF), donde dentistas recién titulados concursan año a año por un cupo para trabajar en algún establecimiento de salud pública primaria a lo largo del país por 3 a 6 años, lo que les da derecho a concursar por una beca de especialidad por 1 a 3 años, beca que incluye el pago de matrículas, aranceles y sueldo. El EDF es una política continua del MINSAL desde hace más de 60 años, que ha permitido que médicos, dentistas, bioquímicos y farmacéuticos puedan ser destinados a zonas alejadas donde normalmente existe poco acceso a este recurso humano. Sin embargo, los dentistas EDF representan una proporción menor de los dentistas contratados por el servicio público, el resto de los dentistas contratados tienen un acceso menor y discontinuo a estas becas. Por último, también existe un programa de becas primarias, en la que son enviados a especializarse y luego deben volver a trabajar al sistema público por el doble del tiempo de duración de la beca cursada ${ }^{19,20}$.

\section{Situación epidemiológica.}

El último estudio enfocado en evaluar el estado de salud bucal a nivel nacional y con financiamiento público fue realizado por Soto et al. en 2006-2007 en población de 12 años ${ }^{21}$. El principal hallazgo de dicho estudio fue un CPOD de 1,9 en esta población, lo que supuso comprobar el logro de la meta de un CPOD de 2,3 planificada para 2010. Sin embargo, una serie de estudios con cobertura local y publicados en revistas científicas nacionales e internacionales han mostrado que la historia de caries es mayor a lo encontrado por Soto et al.

Arias y Fuentes realizaron una revisión sistemática para evaluar la morbilidad bucal en preescolares y escolares de Chile asociado a factores de riesgo en la década 20082018. Para ello, desde una muestra inicial de 304 artículos en las bases de datos SciELO, Scopus, PubMed y WoS, seleccionaron 31 artículos que cumplieron sus criterios. En el caso de preescolares de 2 a 5 años, encontraron que la prevalencia de caries fue de un $23,30 \%$ a un $70,09 \%$, mientras que el ceod mostró valores de 0,79 a 6,83 . En el caso de escolares de 6 a 12 ańos, la prevalencia fue de $33,50 \%$ a $86,64 \%$, mientras que el valor del ceod fue de 1,34 a 5,74 y el de CPOD de 0,15 a 3,1, acá debemos destacar que los estudios que incluyeron solo nińos de 12 ańos mostraron un CPOD de 1,56 a 4,05 y una prevalencia de $63,85 \%$ a $86,64 \%$. En tanto que, los adolescentes de 12 a 17 ańos presentaron una prevalencia de 79,5\% y un CPOD de 1,8 a 9,06 ${ }^{22}$.

Otro de los grupos priorizados por las políticas de salud bucal chilenas, las embarazadas, también presentan una morbilidad bucal alta. Se ha encontrado que tienen hasta un $97 \%$ de necesidad de tratamiento periodontal ${ }^{23} \mathrm{y}$ con valores de CPOD superiores a 8 con una prevalencia de caries activas mayor al $50 \%{ }^{24-26}$, mientras que solo un 35\% de ellas cuenta con buen nivel de conocimientos en salud bucal ${ }^{27}$.

En el grupo de adultos mayores se han reportado CPOD de 22,16, una prevalencia de bolsas periodontales $>5,5 \mathrm{~mm}$ del $20 \%$ y un edentulismo del $7,1 \%$ en Santiago ${ }^{28}$, mientras que en la región de Maule se encontró un CPOD de 25,7, de los cuales 22,4 correspondieron a dientes perdidos y donde el 25,1\% de la muestra eran edéntulos y un 30,1\% requerían de terapia periodontal compleja ${ }^{29}$. En tanto que, en un grupo de adultos mayores postrados se reportó una prevalencia de caries del 100\%, con un COPD de 25,6 y el edentulismo llegó al $46 \%{ }^{30}$. Una encuesta a nivel nacional determinó que el $97,58 \%$ de los adultos mayores presentó al menos un sitio con una pérdida de inserción periodontal $>3 \mathrm{~mm}$, que se redujo a $69,35 \%$ en pérdidas de inserción $>6 \mathrm{~mm}^{31}$. 
El grupo de adultos medios, que ha sido poco priorizado en Chile, presenta también un nivel de morbilidad bucal alto. En un estudio que incluyó adultos de 35-44 años se encontró un CPOD de 15,06 de las cuales 5,9 corresponden a dientes perdidos y dónde el 14,34\% de la muestra tenían menos de 21 dientes ${ }^{32}$. También es importante mencionar los resultados de la Encuesta Nacional de Salud 2016-2017 que se realizó en mayores de 15 años, en esta se encontró que el $27 \%$ de ellos tenía menos de 20 dientes y el $54,6 \%$ tenía al menos una lesión de caries cavitada. Sin embargo, debemos aclarar que la Encuesta Nacional de Salud es realizada por enfermeras, no dentistas ${ }^{33}$.

Por último, la calidad de vida relacionada con salud bucal también ha sido evaluada en población chilena. En el caso de adultos mayores se encontró que el 76,6\% presenta una mala calidad de vida ${ }^{28}$, en tanto que en adolescentes de 11-14 años se encontró que el 68\% reportó que al menos una de sus actividades diarias se vio afectada por problemas bucales ${ }^{34}$. La Encuesta Nacional de Salud 2016-2017 reportó que el 14\% de los mayores de 15 años veían afectada su calidad de vida debido a su salud bucal, esto representa una caída importante desde el 37\% reportado en la Encuesta Nacional de Salud de $2006^{35}$.

\section{Desafíos futuros}

La salud bucal chilena ha visto mejoras considerables en las últimas dos décadas en cuanto a la ampliación de los programas de promoción, prevención y tratamiento odontológico. Estas mejoras han sido desarrolladas sobre la priorización de los grupos infantiles, mujeres y adultos mayores, lo que busca generar cambios positivos de una manera eficiente, acorde a la realidad socioeconómica del país.

Sin embargo, la carga de enfermedades bucales en la población chilena sigue siendo muy alta. Lamentablemente, no se cuentan con encuestas de salud bucal a nivel nacional que cubran a toda la población utilizando la metodología recomendada por la Organización Mundial de la Salud ${ }^{36}$. Si bien las Encuestas Nacionales de Salud incluyen la evaluación del componente odontológico, estás cubren solo a la población mayor de 15 ańos y el examen es realizado por enfermeras ${ }^{33,35}$. A pesar de lo anterior, la percepción es que los indicadores de morbilidad bucal han mejorado notoriamente en las últimas décadas, de la mano de un mejor acceso a atención odontológica. Esto, sin considerar a la población adulta (20 a 60 ańos), la que cuenta con un acceso creciente, pero aun escaso, a tratamiento odontológico.

Ante esta realidad, el Departamento de Salud Bucal del MINSAL ha propuesto un Plan Nacional de Salud Bucal 2018-2030 que aborda la promoción de salud y prevención de enfermedades bucales; provisión de servicios odontológicos de calidad; desarrollo del recurso humano; e investigación, monitoreo y evaluación ${ }^{15}$. Es un programa ambicioso, que considera un enfoque de equidad y los determinantes sociales de la salud, enmarcado en el modelo de atención integral de salud familiar y comunitario.
Sin embargo, en cuanto a investigación, monitoreo y evaluación no existe, de momento, ninguna implementación clara de algún sistema de vigilancia epidemiológica que permita conocer de forma clara y oportuna el estado de salud bucal de nuestra población. Tampoco existe claridad de cuál será el vínculo con las universidades en esta materia, atendiendo a que son ellas quienes, en teoría, cuentan con las capacidades necesarias para desarrollar investigación que aporte al diseño, evaluación y mejoramiento de las políticas públicas en salud bucal.

En relación al desarrollo del recurso humano, la sobreoferta de dentistas, especialmente de generales sin especialidad, es el elefante en la habitación ${ }^{37}$. La sobreoferta de profesionales ha sido ignorada por universidades, el ministerio de educación y el de salud. Ciertamente debe existir una política de mejoras cualitativas y cuantitativas de la fuerza laboral de dentistas en el sector público, pero se debe considerar que existe una gran masa de dentistas generales y especialistas que están subempleados, cesantes y/o desempeñando funciones no odontológicas. Así, no queda claro si el sistema público debe seguir pagando por la formación de especialistas o simplemente contratar a los que ya están disponibles en el mercado. Una cuestión más compleja es si la fuerza laboral odontológica chilena debe estar compuesta solamente por dentistas o se dará espacio a otro tipo de proveedores como los terapeutas dentales, que harían más eficiente el gasto público ${ }^{38}$.

Respecto a la provisión de servicios odontológicos de calidad parece no haber cambios respecto a la priorización de los grupos infantiles, embarazadas y adultos mayores. Si bien algunos adultos jóvenes y medios tienen acceso a tratamiento odontológico, mayormente parcial, todo indica que, durante esta década ese acceso seguirá siendo pobre para quienes tienen de 20 a 60 ańos (el 58,19\% de la población nacional según estimaciones del Instituto Nacional de Estadística). Esto ocurre en el marco de una sobreoferta de dentistas y de más de una treintena de escuelas dentales a lo largo del país ${ }^{37}$, donde de manera creciente se han venido reduciendo la cantidad de procedimientos que deben realizar los estudiantes de odontología debido a la ilógica "falta de pacientes" y donde existen Mesas Intersectoriales de Salud Bucal a nivel nacional y regional con reuniones periódicas de miembros del MINSAL y de las Universidades. En este sentido, sorprende la falta de coordinación para aprovechar la capacidad instalada y la sobreoferta de recurso humano ${ }^{39}$

En tanto que la promoción de salud y prevención de enfermedades bucales se ha centrado en los determinantes sociales de la salud más proximales, específicamente a nivel de estilos de vida. Esta parece una decisión razonable, atendiendo las dificultades para modificar los determinantes más distales o estructurales, sin embargo, ha priorizado estrategias como educación en salud y fluoración. La fluoración del agua potable ha sido implementada de manera progresiva desde $1985^{15}$, por lo que después de 35 años, parece ser que no seguirá reduciendo la prevalencia y severidad de caries en nuestra población. Por otro 
lado, existe claridad que las estrategias tradicionales en salud bucal tienen un impacto escaso y efímero, por lo que no son adecuadas para generar resultados consistentes en el largo plazo ${ }^{40}$. Si bien la inclusión de jardines infantiles y la participación de los padres en el cuidado de la salud bucal de los preescolares aparece como un factor positivo, después de varios años no existe evidencia publicada respecto al impacto que esto ha tenido, la que puede ser escasa si se han seguido usando estrategias tradicionales de educación en salud bucal, centradas principalmente en conocimiento y la prescripción de hábitos.

Por último, aun cuando se menciona, no queda del todo claro cómo se implementará el enfoque de factores comunes para enfermedades crónicas, esto, teniendo especial cuenta del impacto de la dieta en las enfermedades bucales y cardiovasculares ${ }^{41}$. Esta no es una cuestión menor, si consideramos que la mayoría de nuestra población, NNA y adultos, tienen sobrepeso u obesidad ${ }^{33,42}$.

\section{Conclusiones}

La salud bucal chilena ha visto una mejora continua en sus indicadores epidemiológicos y en las coberturas a nivel promocional, preventivo y curativo para diversos grupos de la población. Actualmente, el Ministerio de Salud ha implementado un ambicioso Plan Nacional de Salud Bucal 2018-2030 que busca mejorar el estado de salud bucal y reducir las desigualdades, esto se basa en los conceptos de equidad, intersectorialidad, participación ciudadana e inclusión y no discriminación, y materializa en cuatro ejes estratégicos: promoción de salud y prevención de enfermedades bucales; provisión de servicios odontológicos de calidad; desarrollo del recurso humano; e investigación, monitoreo y evaluación.

A pesar de lo anterior, los estudios muestran que en Chile persisten las inequidades en salud bucal y la población adulta (especialmente hombres) cuentan con escaso acceso a atención odontológica. Esto, en el marco de una sobreoferta alarmante de dentistas que se titulan ańo a año en el país.

Se hace necesario una mayor integración de la red de salud con las universidades, de manera que estas últimas pongan a disposición su capacidad de atención clínica y desarrollo de investigación al servicio de las necesidades en salud bucal de la población chilena.

\section{Referencias bibliográficas}

1. Martin MP, Alfaro J. Políticas de bienestar en contextos neoliberales: tensiones del modelo chileno. Caderno CRH 2017;30(79):137-155.

2. Artaza O, Méndez CA. Crisis social y política en Chile: la demanda por acceso y cobertura universal de salud. Rev Panam Salud Publica 2020;44:e16.

3. del Romero L. Cartografías de la desigualdad: una década de conflictos de vivienda y nuevas resistencias en Santiago de Chile. Análisis del conflicto de la Maestranza de San Eugenio. EURE (Santiago) 2018;44(132):47-66.
4. Lunecke A. Inseguridad ciudadana y diferenciación social en el nivel microbarrial: el caso del sector Santo Tomás, Santiago de Chile. EURE (Santiago) 2016;42(125):109129.

5. Santos H, Elacqua G. Segregación socioeconómica escolar en Chile: elección de la escuela por los padres y un análisis contrafactual teórico, Rev CEPAL 2016;119:133-148.

6. Becerril-Montekio Víctor, Reyes Juan de Dios, Manuel Annick. Sistema de salud de Chile. Salud Pública Méx. 2011;53(Suppl 2): s132-s142.

7. Goic A. El Sistema de Salud de Chile: una tarea pendiente. Rev Méd Chile 2015;143(6):774-786.

8. Superintendencia de Salud. ¿Cómo se clasifican los beneficiarios de Fonasa según su tramo? [Consultado el 20 de febrero de 2020]. Disponible en: http://www.supersalud. gob.cl/consultas/667/w3-article-6304.html

9. Cid C, Uthoff A. La reforma a la salud pendiente en Chile: reflexiones en torno a una propuesta de transformación del sistema. Rev Panam Salud Pública 2018;41:e170.

10. García-Huidobro D, Barros X, Quiroz A, Barría M, Soto $\mathrm{G}$, Vargas I. Modelo de atención integral en salud familiar y comunitaria en la atención primaria chilena. Rev Panam Salud Pública 2018;42:e160.

11. Urriola C, Infante A, Aguilera I, Ormeño H. La reforma de salud chilena a diez años de su implementación. Salud Pública Mex. 2016;58:514-521.

12. MINSAL. Ley Ricarte Soto. [Consultado el 20 de febrero de 2020]. Disponible en: https://www.minsal.cl/ leyricarte/

13. Servicio de Salud Nuble. Indicadores de Actividad de Atención Primaria. [Consultado el 20 de febrero de 2020]. Disponible en: http://www.serviciodesaludnuble. $\mathrm{cl} /$ sitio/compromisos-e-indicadores-de-gestion-en-salud/ indicadores-de-actividad-de-atencion-primaria/

14. MINSAL. Salud Bucal. [Consultado el 20 de febrero de 2020]. Disponible en: https://www.minsal.cl/salud-bu$\mathrm{cal} /$

15. MINSAL. Plan Nacional de Salud Bucal 2018-2030. Santiago: MINSAL; 2017.

16. Jara G, Werlinger F, Cabello R, Carvajal P, Espinoza I, Giacaman R, Lee X, Morales A, Arteaga O, Gamonal J. Synopsis of the Oral Health Situation in Chile - Part I: Explicit Guarantees and Clinical Guidelines. Rev Clin Periodoncia Implantol Rehabil Oral 2018;11(3):187190.

17. Nenen A, Courdurier C, Arcos A. Riesgo de Caries en Niños que Ingresan al Programa de Población en Control con Enfoque de Riesgo Odontológico. Int J Odontostomat. 2019;13(4):437-441.

18. Cornejo-Ovalle M. Atención Dental FONASA Libre Elección... para quien pueda pagar. J Oral Res. 2014;3(1):11-12.

19. Cartes-Velásquez R. Percepción de la capacitación a Dentistas Generales de Zona: encuesta nacional. Rev Chi Salud Pública. 2011;15(2):77-82. 
20. Cartes-Velásquez R. Caracterización de los Postulantes a Dentista General de Zona. Int J Odontostomat. 2011;5(1):71-76.

21. Soto L, Tapia R, Jara G, Rodríguez G, Urbina T, Venegas C. Diagnóstico nacional de salud bucal del adolescente de 12 años y evaluación del grado de cumplimiento de los objetivos sanitarios de salud bucal 2000-2010. Santiago de Chile: Facultad de Odontología, Universidad Mayor; 2007.

22. Arias A, Fuentes L. Morbilidad bucal y factores asociados en preescolares y escolares chilenos durante la última década. Revisión sistemática. Concepción: Universidad Andrés Bello; 2018.

23. Norambuena-Suazo P, Palma-Cárdenas I. Necesidad de tratamiento periodontal en embarazadas, Frutillar, Chile, 2015. Rev Clin Periodoncia Implantol Rehabil Oral. 2016;9(2):121-124.

24. Sabando V. Factores de riesgo de caries en embarazadas beneficiarias de Centros de Atención Primaria la Comuna de Quillota y Calera, Chile: estudio transversal. Odontol Vital. 2015;2(23):17-22.

25. Traeger RLM. Prevalencia y severidad de caries dental en embarazadas atendidas por GES" salud oral integral", en un centro de salud primaria, comuna de Punitaqui, IV región de Coquimbo, año 2013. Santiago de Chile: Universidad Finis Terrae; 2014.

26. San-Martín HV, Wiliams DC, Tsukame KY, Carstens AM, Coloma OMC, Lorenzo VM, Arenas VG. Comparacion de la salud oral de embarazadas y puerperas hospitalizadas segun uso de programa de salud oral integral de la embarazada: Estudio transversal. Int J Odontostomat. 2018;12(2):110-116.

27. Núñez J, Moya P, Monsalves MJ, Landaeta S. Nivel de conocimiento de salud oral y utilización de GES odontológico en puérperas atendidas en una clínica privada, Santiago, Chile. Int J Odontostomat. 2013;7(1):39-46.

28. Moya P, Chappuzeau E, Caro JC, Monsalves MJ. Situación de salud oral y calidad de vida de los adultos mayores. Rev Estomatol Herediana. 2012;22(4):197-202.

29. Mariño R, Giacaman RA. Factors related to unmet oral health needs in older adults living in Chile. Arch Gerontol Geriatrics. 2014;58(3):454-459.

30. Quinteros ME, Nuñez L. Salud oral en adultos mayores postrados. Int J Odontostomat. 2014;8(3):337-343.
31. Gamonal J, Mendoza C, Espinoza I, Munoz A, Urzua I, Aranda W, Carvajal P, Arteaga O. Clinical attachment loss in Chilean adult population: first Chilean national dental examination survey. J Periodontol. 2010;81(10):1403-1410.

32. Urzua I, Mendoza C, Arteaga O, Rodríguez G, Cabello $\mathrm{R}$, Faleiros $\mathrm{S}$, et al. Dental caries prevalence and tooth loss in chilean adult population: first national dental examination survey. Int J Dent. 2012;810170. DOI: $10.1155 / 2012 / 810170$

33. MINSAL Encuesta Nacional de Salud 2016-2017. Santiago de Chile: MINSAL; 2017.

34. Moreno X, Vera C, Cartes-Velásquez, R. Impacto de la salud bucal en la calidad de vida de escolares de 11 a 14 años, Licantén, 2013. Rev Clin Periodoncia Implantol Rehabil Oral. 2014;7(3):142-148.

35. MINSAL Encuesta Nacional de Salud 2006. Santiago de Chile: MINSAL; 2006

36. World Health Organization. Oral health surveys: basic methods. Geneva: World Health Organization; 2013.

37. Cartes-Velasquez RA. Exponential growth of dental schools in Chile: effects on academic, economic and workforce issues. Braz Oral Res. 2013;27(6):471-477.

38. Hill H, Macey R, Brocklehurst P. A Markov model assessing the impact on primary care practice revenues and patient's health when using mid-level providers, lesson learned from the United Kingdom. J Public Health Dent. 2017;77(4):334-343

39. Cartes-Velásquez R. Reflexiones en torno a la educación odontológica en Chile. Pensar Salud. 2013;1(1):6-7.

40. Stein C, Santos NML, Hilgert JB, Hugo FN. Effectiveness of oral health education on oral hygiene and dental caries in schoolchildren: Systematic review and meta-analysis. Community Dent Oral Epidemiol. 2018;46(1):30-37.

41. Chomitz VR, Park HJ, Koch-Weser S, Chui KKH, Sun L, Malone ME, et al. Modifying dietary risk behaviors to prevent obesity and dental caries in very young children: results of the Baby Steps to Health pediatric dental pilot. J Public Health Dent. 2019;79(3):207-214.

42. Castro M, Muros JJ, Cofré C, Zurita F, Chacón R, Espejo $\mathrm{T}$. Índices de sobrepeso y obesidad en escolares de Santiago (Chile). J Sport Health Res. 2018;10(2):251-6. 